ZUÑIGA, V. M. E. (2018) Caso: diseño e implementación de la estrategia de intervención social en una institución micro financiera en la Ciudad de Cali-Colombia, con un modelo institucional orientado en temas de protección y capacitación de los servicios no financieros. Cafi, v. 1 n. 1, p. 58-71

\title{
CASO: DISEÑO E IMPLEMENTACIÓN DE LA ESTRATEGIA DE INTERVENCIÓN SOCIAL EN UNA INSTITUCIÓN MICRO FINANCIERA EN LA CIUDAD DE CALI -COLOMBIA, CON UN MODELO INSTITUCIONAL ORIENTADO EN TEMAS DE PROTECCIÓN Y CAPACITACIÓN DE LOS SERVICIOS NO FINANCIEROS.
}

\section{CASE: DESIGN AND IMPLEMENTATION OF THE SOCIAL INTERVENTION STRATEGY IN A MICRO FINANCIAL INSTITUTION IN THE CITY OF CALI- COLOMBIA, WITH AN INSTITUTIONAL MODEL ORIENTED IN PROTECTION AND TRAINING ISSUES OF NON-FINANCIAL SERVICES.}

Recebido: 16/11/2017 - Aprovado: 21/12/2017 - Publicado: 02/01/2018
Processo de Avaliação: Double Blind Review

Victor Manuel Escobar Zuñiga ${ }^{1}$

\section{RESUMEN}

La ponencia busca mostrar un estudio de caso, que tiene como objetivo, el resultado de un modelo de educación financiera, diseñado he implementado en una institución micro financiera, con una estrategia focalizada de intervención social a población vulnerable, fundamentado en mejorar la calidad de vida de las personas en la sociedad, especialmente las que realizan actividades microempresariales y no cuentan con disponibilidad de recurso económicos. Finalmente en este trabajo se presenta un resumen de las teorías microfinancieras internacionales, que soportaron el estudio conceptual del modelo de educación citado en el párrafo anterior, adicionalmente se identifica la metodología utilizada y los resultados de las diferentes etapas investigativas que condijeron al estudio de caso investigativo, como lo fue el desarrolló un estudio piloto que dio como respuesta, una línea de orientación del doctor financiero y de implementación, articulada con las fases que se relacionan a la caracterización social demográfica de la población de Cali y un diagnóstico de la situación financiera que se encontró entre los clientes que asistieron a la asesoría en el consultorio financiero.

Palabras Claves: Economía, Microfinanciera, Educación Financiera

\section{ABSTRACT}

The paper seeks to show a case study, which aims, the result of a financial education model, designed I have implemented in a microfinance institution, with a focused strategy of social intervention to vulnerable population, based on improving the quality of life of people in society, especially those who carry out microenterprise activities and do not have availability of economic resources. Finally, this paper presents a summary of international microfinance theories, which supported the conceptual study of the education model cited in the previous paragraph, additionally identifies the methodology used and the results of the different investigative stages that led to the investigative case study, as it was he developed a pilot study that gave as response, a line of orientation of the financial and implementation doctor, articulated with the phases that relate to the demographic social characterization of the population of Cali and a diagnosis of the financial situation that He was among the clients who attended the counseling in the financial office.

Keywords: Economy, Microfinance, Financial Education

\footnotetext{
${ }^{1}$ Docente de Plata EMAVI FAC; Administrador de Empresas; Especialista en Administracion Publica; Magister en Politica Publica; Universidad del Valle; Doctorado en Proyectos Ac tualmente; Universidad Internacional Iberoamericana de Mexico; vmescobar@emavirtual.edu.co
} 


\section{INTRODUCCIÓN}

La experiencia de una Institución Financiera de la ciudad de Cali especializada en el microcrédito, en productos de micro finanzas, en la intervención social y en el tema de educación financiera, en contraste con los componentes principales que debe tener un Programa de Administración, como lo son las competencias Organizacionales, Económicas Y financieras sirvió como base para la investigación que da origen a esta ponencia. Esta práctica fue valiosa porque partió de la necesidad de generar condiciones para mejorar la situación económica de la población con menos ingresos económicos, ya que las microfinanzas han sido vistas como un mecanismo efectivo para promover la bancarización y el acceso a capital. Según los datos evidenciados en la última década, esto ha sido efectivo, ya que ha aumentado la tasa de bancarización, siendo el microcrédito el producto estrella, sobre el cual se acentúa la presente reflexión, dado que la masificación es un beneficio para el sistema financiero y no para el usuario, teniendo en cuenta sus altos costos y desequilibrios.

Dentro de la política pública de bancarización se impulsa el fortalecimiento de las micro finanzas, y el marco normativo como el que se creó con la Ley 1328 de 2009, que obliga a desarrollar aspectos como la Educación Financiera en favor de un mayor y mejor uso de los productos financieros. Partiendo de estos principios, la Institución MF proyectó implementar dentro de sus programas estratégicos la educación financiera como un tema institucional, generando un impacto significativo entre sus clientes, considerando que estos se ubican en sectores de la ciudad con más bajos ingresos económicos.

La Institución MF diseñó una propuesta de intervención social que inició con la definición de la mejor estrategia, después de varios análisis se concretó en un modelo de educación financiera que recurre al consultorio financiero y por supuesto al contacto directo con los usuarios de esta clase de productos.

En la primera parte de los resultados se muestra cómo se consolidó la propuesta financiera, en el contexto en el cual se hace la descripción del proceso de intervención ejecutado en la ciudad de Cali. También se definen los alcances y lineamientos que debe tener la propuesta y adicionalmente se realiza el análisis respetivo sobre el modelo de intervención implementada en el respectivo estudio de caso.

Posteriormente se hace una caracterización de la constitución de la estrategia local de intervención, considerando las condiciones de la población beneficiada, es decir los clientes de la institución. A continuación, se presenta el desarrollo de la etapa denominada pilotaje donde 
se hace una valoración para definir qué método es el más idóneo para atender la población objetivo y los temas que deben ser tratados durante las asesorías. Para lograr esto se recurrió a consultar con aliados estratégicos, con personal interno de la organización, y una muestra representativa de clientes, usando para ello entrevistas y grupos focales. Finalmente se llegó a la conclusión que el método más efectivo era el consultorio financiero atendidos por expertos en el tema de educación financiera.

En la última parte se presentan los resultados de la etapa de implementación, en esta fase se relacionan la caracterización social demográfica de la población y un diagnóstico de la situación financiera que se encontró entre los clientes que asistieron a la asesoría en el consultorio financiero.

El consultorio financiero demostró ser un método efectivo para lograr el desarrollo de la educación financiera, para que los clientes hagan mejor y mayor uso de los productos de micro finanzas. Lo visto a lo largo de la experiencia de la Institución MF podrá aplicarse en otras instituciones que ofrezcan toda clase de productos financieros, siempre que se respete el objetivo que se propone el Estado en lo que corresponde a la Política Pública y los diferentes Programas Sociales como : Protección al Consumidor Financiero, Bancarización e Inclusión Financiera, Responsabilidad Social y Protección a la Población Vulnerable y donde primen los intereses compartidos, sin el agresivo y maquillado asistencialismo del capitalismo salvaje.

Para el desarrollo de los objetivos del estudio de caso se planteó (1) un Objetivo General y (4) cuatro Objetivos Específicos

\subsection{GeneraL}

- Diseñar de un modelo de educación financiera para una Institución microfinanciera orientado en temas de protección y capacitación de los servicios no financieros que dan respuesta a una ley en la ciudad de Cali (Colombia).

\subsection{ESPECÍFICOS}

- Describir el modelo teórico actual de educación financiera que se implementa la Institución Microfinanciera.

- Caracterizar y analizar la estrategia local en relación a la población beneficiada.

- Modelar las estrategias de educación financiera al contexto de la Institución microfinanciera para el desarrollo de una prueba piloto.

- Describir los principales resultados obtenidos en el trabajo etnográfico sobre el pilotaje 


\section{REVISIÓN DE LITERATURA}

La norma de protección de los diferentes consumidores financieros es regulada por el Estado en todos los países del mundo, para el caso de Colombia es normativa, y está regulada por la Superintendencia Financiera bajo el marco de la Ley 1328 de 2009, denominada Ley de Protección al Consumidor Financiero. Este organismo oficial exige a las entidades velar por los derechos de los usuarios del sistema financiero y las competencias de las instituciones frente a la implementación de herramientas que permitan a los consumidores el uso racional y eficiente de los productos y servicios bancarios, como lo son las micro-financieras, dado que estas trabajan con población vulnerable dando acceso a servicios que, en su momento, la banca tradicional no consideraba importante y, por tanto su enfoque estaba orientado a población con capacidad de pago y no a personas deficitarias por el riesgo que estas representaban para su estado de ingresos y resultados. Al respecto, Rodríguez G. (2008) en su artículo sobre El impacto de las microfinanzas, resultado de algunos estudios, con énfasis en el sector financiero, argumenta que el impacto de este sector de las finanzas es un tema inacabado. Plantea que las posiciones de varios autores investigados han coincidido en que los movimientos microfinancieros todavía tienen pendiente afrontar el reto de clarificar el efecto de las microfinanzas y hace especial énfasis en el microcrédito en particular y en la reducción de la pobreza. Aunque, Rodríguez reconoce que hay investigaciones sobre el tema, manifiesta que han sido aisladas, ya que se enfocan en un sólo tipo de impacto y no se estructuran de tal manera que puedan percibirse desde una mirada global y con perspectivas de análisis completos, e incluso, por qué no, con un referente comparativo en diferentes latitudes.

Un aporte importante sobre el tema de las microfinanzas y el desarrollo social de las mismas, lo hace Otero, M. (1998). El nuevo mundo de las finanzas microempresariales: estructuración de instituciones financieras sanas para los pobres. En su investigación evidencian las diferentes experiencias de crédito y metodologías probadas de servicios financieros integrales para trabajar con población vulnerable mexicana, adicionalmente en ella se refleja el fenómeno que presentan las IMF, las cuales tienen el peso de trabajar arduamente con población con insuficiencia económica. En su efecto, las experiencias referidas por la investigadora en este texto guía, proporcionan herramientas fundamentales para el abordaje asertivo de casos con personas excluidas del sector financiero por las características intrínsecas de este grupo social. 
Sobre la anterior lectura, Vásquez M. (1997) Director del Institutito Saldebas., argumenta que México estaba pasando por una situación muy complicada, dado que la banca tenía problemas de liquidez por el mal manejo de la relación de los créditos otorgados a la población de escasos recursos, sumado al carente diseño de programas y metodologías que respondieran a ese fenómeno; por ende la reflexión teórica del texto de la autora citada, Otero (1998) invitaba a afinar las metodologías de trabajo de las diferentes ONG que abordaban las microfinanzas, con el fin de construir organizaciones auto sostenibles que permitieran otorgar créditos a los más necesitados, con programas de protección como el ahorro y las bondades de hacer un uso adecuado del dinero mismo, con el fin de mejorar la calidad de vida de las personas y de las mismas ONG. De ahí que surja la importancia de trabajar en políticas incluyentes que beneficien a la población menos favorecida, dado que la carencia de estas repercute en la creación y sostenimiento de modelos informales de financiamiento para la satisfacción de necesidades individuales tales como prestamistas, tiendas de prenda, agiotistas entre otros. Fenómeno que conlleva problemas colaterales como el sobreendeudamiento y el despojo de haberes en casos de incumplimiento de los pagos pactados. Sobre este aspecto, Gómez Z. (2012) consideran que el fortalecimiento de las capacidades financieras de la población, representa una herramienta fundamental para mejorar los niveles de inclusión financiera y el desarrollo de actitudes y comportamientos asertivos frente al manejo del dinero, la planeación de su futuro, el aprovisionamiento ante eventos esperados e inesperados, escogencia de productos y búsqueda de información pertinente.

Otro estudio que aborda esta temática es el realizado por el sociólogo chileno D. Cavadas (2002), en su investigación denominada Evaluación del área del Microcrédito un Techo para Chile, muestra el resultado de una política orientada a la reducción de la pobreza en el mundo. Los planteamientos metodológicos sugeridos en el estudio, fueron aplicados en la institución "Un Techo para Chile", con el fin de iniciar un programa de microcrédito para el sector agrario. Adicionalmente, la investigación aporta el análisis de estudios multilaterales de otras instituciones, tales como La FAO: Organización de las Naciones Unidas para la Agricultura y la Alimentación, "Microcrédito: efectos en la pobreza rural y el medio ambiente", sobre el estado mundial de la agricultura y la alimentación realizado en Roma, marzo del 2000). De lo anterior se identifica los trabajos realizados por 1 el autor Aroca P. (2002) quien realizó un estudio detallado sobre la aplicabilidad de programas de microcrédito en el sector rural en países como Brasil y Chile. 
Es pertinente mencionar que Cavada, destaca las numerosas investigaciones de la FAO en temas de microcrédito en el campo rural, con sus efectos en temas económicos y sociales, fundamentados en procesos de capacitación y adiestramiento financiero y microempresarial; además del fomento de los servicios no financieros.

El énfasis de los trabajos citados anteriormente, se centra en la importancia de la labor social respecto a la educación financiera, y va más allá del simple hecho de ampliar la cobertura en cuanto a productos financieros. Ciertamente, en los distintos trabajos se ratifica la importancia de trabajar sobre los productos sociales que deben tener las instituciones microfinancieras promovidas por las ONG y el sector formal, para ofrecer a las personas vulnerables orientación en temas de protección frente al manejo de sus finanzas personales y al uso responsable de productos financieros. Sobre el particular, Cavadas, afirma que "los resultados estarían dados en reforzar la capacidad de ahorro de los prestatarios y, en último término, su sentido de la responsabilidad frente al cumplimiento de los pagos de la deuda. Es por todo ello que los efectos del microcrédito pueden ser no sólo de índole económico, sino también social pg. 2, 8, 9".

Otras de las investigaciones que refuerzan este referente investigativo son los estudios realizados por Ostrom, y Walker (1994) estos se fundamentan en los efectos sociales del trabajo de las instituciones microfinancieras frente al fenómeno de colectividad de los individuos. Los análisis evidencian que si las microfinancieras cuentan con un marco institucional acorde para informar y comunicar, las personas de escasos recursos estarían en capacidad de cooperar tomando decisiones que aumenten la productividad. Por lo tanto, las organizaciones microfinancieras apelan a la garantía colectiva de los deudores, contrarrestando otro de los factores que afecta la calidad de vida de las personas, el sobreendeudamiento causante de la morosidad. Al respecto, la autora, Muñoz, M. (2007) escribió un artículo llamado: La eficiencia del personal en las microfinancieras influye en la cartera vencida, la investigación trabaja una serie de variables que afectan el normal desarrollo y la eficiencia de las diferentes micro financieras. Muñoz refiere que estas pueden estar definidas por el número de clientes atendidos por empleado; sin embargo, cuando se tienen deficiencias en la capacitación o en el manejo de la información y carencia de objetividad por la falta de profesionalismo del personal institucional, no se cuenta con la institucionalización adecuada y entonces, " se incurre en un incremento en el número de acreditados atendidos por empleado, lo cual puede ocasionar que se pierda el control de la cartera deteriorando el fondeo de la calidad de la cartera”. De acuerdo al planteamiento de la autora, en ocasiones las personas que emplean en las distintas entidades 
financieras, trabajan bajo la presión de cumplir metas económicas, o cuotas de venta lo que origina una de las causas de las malas colocaciones de las carteras, es decir, ofrecen productos financieros a clientes sin que estos últimos tengan la capacidad de pago que le permita responder a la obligación adquirida.

En esta línea de análisis, sobre el profesionalismo en la venta de servicios microfinancieros, la autora Olivares (1989) con su obra "Una metodología de trabajo con el sector informal”, aporta una síntesis sobre los aspectos metodológicos más significativos de la labor de la organización "Acción Internacional” en el campo del desarrollo microempresarial. Olivares aborda asuntos importantes para los profesionales que laboran en el sector informal y sugiere una serie de acercamientos basados en lecciones aprendidas por medio de la experiencia, aproximaciones tales como el conocimiento etnográfico y la capacitación de grupos focales.

En suma, los estudios convergen en que los sistemas financieros pueden generar mayor impacto social en la medida en que se trabaje con herramientas metodológicas, que faciliten la mejora en la calidad de vida de las personas de bajos ingresos o pertenecientes a la economía informal. De acuerdo a lo planteado por los autores citados, el desarrollo y la sostenibilidad de una política microfinanciera, es exitoso si está orientada hacia temas de capacitación a población vulnerable sobre temas financieros contextualizados, repercutiendo en la constitución de una banca socialmente responsable y que provea mecanismos de intervención diferenciado de acuerdo a los datos arrojados en los análisis de las condiciones etnográficas del mercado.

\section{METODOLOGÍA}

La metodología Utilizada en el Estudio de Caso comenzó definiendo la Unidad de Análisis que para estudio de Caso fue El Pilotaje del Consultorio Financiero de IMF (Institución Microfinanciera) por ser el órgano Técnico, Coordinador y Direccionador del proceso en el sur occidente colombiano en el año 2011 y 2012. En este se evaluaron tres aspectos, 
Tabla I Nivel de Análisis

\begin{tabular}{|l|l|l|}
\hline \multicolumn{2}{|c|}{ NIVEL DE ANALISIS } \\
\begin{tabular}{|l|l|} 
Aspectos de \\
participación
\end{tabular} & $\begin{array}{l}\text { Respuesta participativa de los actores } \\
\text { líderes del proceso }\end{array}$ & $\begin{array}{l}\text { Instrumentos de participación utilizados } \\
\text { por los actores, decisiones, } \\
\text { aprovechamiento y apoyo institucional }\end{array}$ \\
\hline $\begin{array}{l}\text { Aspectos de } \\
\text { organización }\end{array}$ & $\begin{array}{l}\text { Planeación y Organización y voluntad } \\
\text { institucional, estructura administrativa y } \\
\text { operativa }\end{array}$ & $\begin{array}{l}\text { Actores involucrados y Numero de } \\
\text { Comités, talleres de conformación de ejes } \\
\text { temáticos, grupos focales }\end{array}$ \\
\hline $\begin{array}{l}\text { Aspectos de } \\
\text { priorización y } \\
\text { eficiencia }\end{array}$ & $\begin{array}{l}\text { Reconocimiento social, Político y } \\
\text { Económico a partir de los beneficios a } \\
\text { obtener y la participación equitativa en los } \\
\text { procesos decisorios de priorización. }\end{array}$ & $\begin{array}{l}\text { Proyectos concretos y aceptados por los } \\
\text { actores involucrados en el proceso }\end{array}$ \\
\hline Fuente: datos de la investigación (2017). & \\
\hline
\end{tabular}

\subsection{ESTRATEGIA DE INVESTIGACIÓN}

La estrategia a utilizar de acuerdo a la realidad de los servicios de protección social, en relación a la oferta institucional financiera y su fenómeno microfinancieros, fue el estudio de caso.

\subsection{Muestreo y Fuente de Datos}

El tipo de muestra para el análisis fue un muestreo teórico o intencionado del Consultorio Financiero de una institución microfinanciera en la ciudad de Cali por ser el órgano que coordina el proceso, éste representó el universo. Los datos a recoger fueron cuantitativos y cualitativos, representados en grupos focales, análisis documental, observación de los participantes y registro de archivos. En la fase de pilotaje se consultaron para sensibilización 196 personas, de las cuales 108 recibieron la atención del Dr. Financiero, en esta etapa se evalúo su situación financiera acorde a los parámetros de la educación financiera.

\subsection{TIPO DE ESTUDIO}

Investigación de tipo descriptivo.

\subsection{Métodos de Procesamiento de Información y ANÁlisis}

La estrategia del estudio es fue una estrategia narrativa, por la precisión de los datos desde el nivel descriptivo, apoyándose también en una estrategia de descomposición temporal, que permitiera un examen más explícito de la información procesada etc. 


\subsection{MÉTODOS DE CONTROL Y VALIDEZ DE LA INFORMACIÓN}

Los métodos de control y validez que se utilizaron para la investigación son los estándares de evaluación y principios que muestran la factibilidad, corrección, precisión y utilidad de la investigación

\section{RESULTADOS}

\section{Caracterización del proceso de intervención social realizado por la institución} microfinanciera entre los usuarios de microfinanzas en la ciudad de santiago de cali.

en la institución MF para lograr desarrollar un programa de educación financiera dentro del esquema de responsabilidad de esta organización y del modelo que propone ASOBANCARIA que agrupa a las instituciones de carácter financiero del país (Colombia) Entre los usuarios de microfinanzas en la ciudad de Santiago de Cali.

Descripción del proceso de intervención: La propuesta de intervención que presentó la institución MF para desarrollar un programa de educación financiera se hizo considerando cuatro grandes fases, tal como se relaciona en la tabla (II) como se observa cada fase contiene un objetivo y las tareas que los hacen posibles.

Tabla II (Propuestas de Intervención)

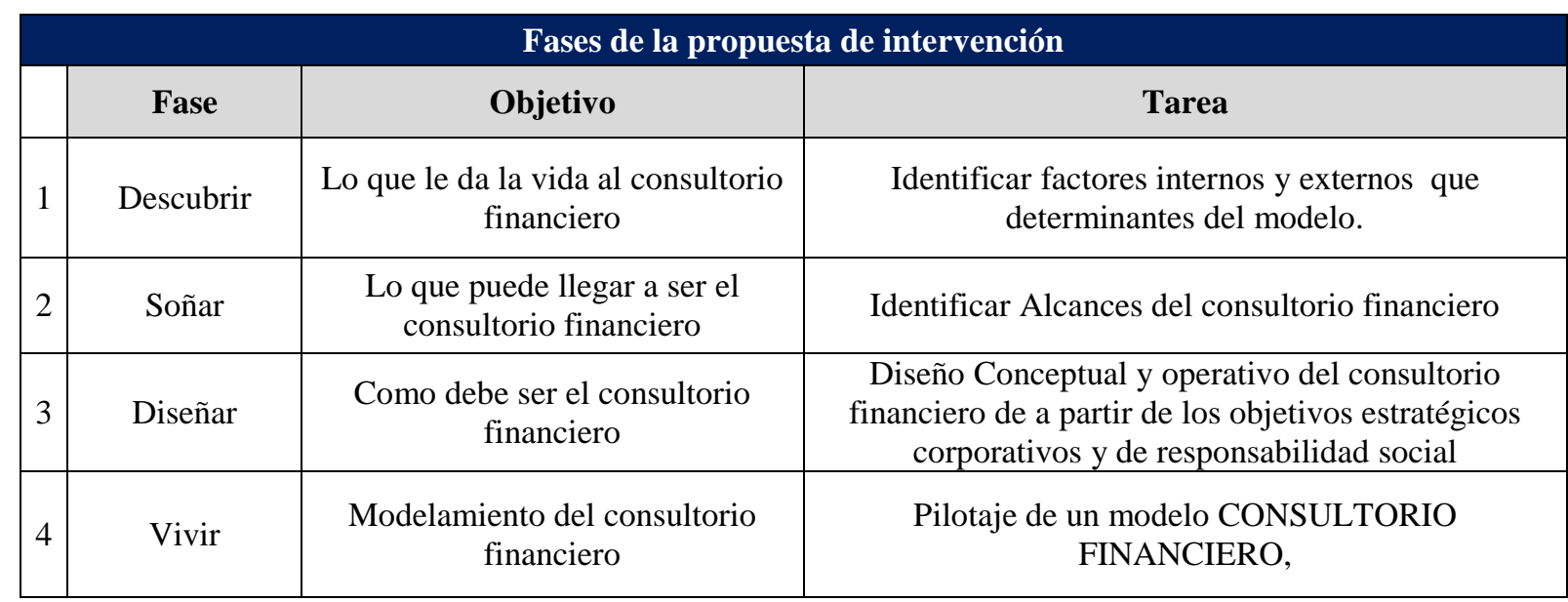

Fuente: datos de la investigación (2017).

\section{Estrategias utilizadas para la implementación de los procesos de intervención de la estrategia local.}

Considerando el contexto donde existe una creciente demanda de servicios financieros, se hace necesario profundizar en el uso de los servicios de educación financiera que hace parte de la visión de Responsabilidad Social Empresarial de las Instituciones financieras, y que es 
algo obligatorio según lo dispone la Ley 1328 de 2009. En el contexto de la Institución MF se propusieron unos objetivos para la estrategia de intervención social que se dará en el contexto local. Para el desarrollo de la estrategia de intervención se desarrolló el Modelo Conceptual

\section{de Consultorios Financieros (grafica 1)}

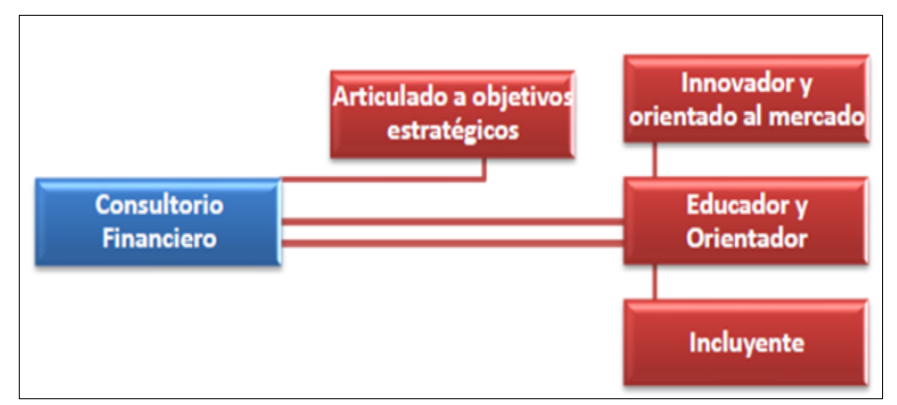

Grafica 1 Modelo Consultorios Financieros

Fuente. ESCOBAR ZUÑIGA Víctor Manuel \& QUIÑONEZ RIZO. Edgar Proyecto Consultorio Financiero. Informe Final. Consultor Aso bancaria. Diciembre 2012

Resultados obtenidos en el trabajo etnográfico sobre el pilotaje y la fase de implementación.

Tabla III Muestra de pilotaje del consultorio financiero

\begin{tabular}{c|c}
\hline CONSOLIDADO & TOTAL \\
\hline TOTAL PERSONAS SENSIBILIZADAS & 78 \\
\hline TOTAL CITAS ASIGNADAS & 49 \\
\hline PERSONAS ATENDIDAS HASTA 1 SESION & 4 \\
\hline PERSONAS ATENDIDAS HASTA 2 SESION & 12 \\
\hline PERSONAS ATENDIDAS HASTA 3 SESION & 24 \\
\hline TOTAL PERSONAS ATENDIDAS & 40 \\
\hline TOTAL PERSONAS ATENDIDAS POR SERVICIO DE ASESORIA FINANCIERA & 39 \\
\hline TOTAL PERSONAS ATENDIDAS POR SERVICIO DE INFORMACION FINANCIERA & 1 \\
\hline TOTAL CITAS PENDIENTES & 25 \\
\hline TOTAL INASISTENCIAS & 7 \\
\hline TOTAL ASISTENCIA A 1 SESION & 40 \\
\hline TOTAL ASISTENCIA A 2 SESION & 36 \\
\hline TOTAL ASISTENCIA A 3 SESION & 24 \\
\hline CASOS CANCELADOS & 2 \\
\hline
\end{tabular}

Fuente. Fuente. ESCOBAR ZUÑIGA Víctor Manuel, según datos de la gerencia general de la Institución MF. Cali. 2013

En la etapa de pilotaje se realizó el contacto con los usuarios de los productos financieros de la Institución MF, para lo cual se siguió el proceso ilustrado en la Tabla (III), donde se inició con una sensibilización y se terminó con una ruta de apoyo.

En éste objetivo del estudio de Caso, se hizo una caracterización de los resultados que se obtuvieron de la prueba piloto que hizo la Institución financiera MF que se realizó en el año 2012. Durante este periodo de coloco en funcionamiento el consultorio financiero para la atención de los clientes. 
En un principio la Institución Financiera MF estaba trabajando en dos líneas base de intervención la primera; el Dr. Financiero (En esta se da orientación financiera ) y la segunda de información financiera (En esta se orientaba a las personas sobre los diferentes temas de Educación financiera y productos del mercado financiero) dada la demanda de tiempo que requería la última con respecto a temas investigativos sobre el sector financiero y la poca demanda del mismo, se decidió trabajar sobre una línea base como la del Dr. Financiero que se consideró un éxito total por el interés de los clientes atendidos,

\section{Resultados cuantitativos}

En la Tabla III se presenta la síntesis de los resultados cuantitativos, donde se detalla la cobertura del programa, para el caso de las personas atendidas por el servicio de asesoría financiera, se atendieron 114 personas.

Tabla III implementación del pilotaje del Consultorio Financiero

\begin{tabular}{|c|c|c|}
\hline CONSOLIDADO & TOTAL & $\%$ \\
\hline TOTAL PERSONAS SENSIBILIIADAS & 196 & $100 \%$ \\
\hline TOTAL CITAS ASIGNADAS & 178 & $91 \%$ \\
\hline PERSONAS ATENDIDAS CON 1 SESION & 73 & $64 \%$ \\
\hline PERSONAS ATENDIDAS CON 2 SESIONES & 18 & $16 \%$ \\
\hline PERSONAS ATENDIDAS CON 3 SESIONES & 23 & $20 \%$ \\
\hline TOTAL PERSONAS ATENDIDAS & 114 & $64 \%$ \\
\hline TOTAL PERSONAS ATENDIDAS POR SERVICIO DE ASESORIA FINANCIERA & 108 & $95 \%$ \\
\hline TOTAL PERSONAS ATENDIDAS POR SERVICIO DE INFORMACION FINANCIERA & 6 & $5 \%$ \\
\hline TOTAL CITAS PENDIENTES & 0 & 0 \\
\hline TOTAL INASISTENCIAS A CITAS & 64 & $36 \%$ \\
\hline CASOS CANCELADOS & 2 & $1 \%$ \\
\hline
\end{tabular}

Fuente. ESCOBAR ZUÑIGA Víctor Manuel. Proyecto Consultorio Financiero. Informe Final. Consultor Aso bancaria. Diciembre 2012.

Durante la implementación del pilotaje del Consultorio Financiero se propuso como meta atender a 100 personas, ésta se superó, la cifra real fue de 114 clientes atendidos, las cuales hacen referencia al 100\% de la población total, el cronograma de atención por mes fue el siguiente:

En el mes de junio (2012) se atendió las primeras dos (2) personas que representan un $2 \%$, siendo este el mes de menor afluencia de público dado que apenas se estaba iniciando y no estaban definidos los procesos aun, en comparación con el mes de diciembre que si se tenía un proyecto bien estructurado y con un espacio físico de trabajo adecuado para esta época, el resultado fue diferente, dado que se atendió el $41 \%$ de la población total del pilotaje correspondiente a 47 personas atendidas. Los meses de Julio con 22 pacientes (19\%), Septiembre con 9 pacientes (8\%), Octubre 5 pacientes (4\%), y Noviembre 29 pacientes $(25 \%)$ contribuyeron para lograr la meta propuesta en esta etapa de pilotaje. 
La focalización del consultorio financiero estuvo orientada entre el cliente interno y el cliente externo (Instituciones) la ruta interna que se articuló con el consultorio en el año 2012 estuvo compuesta por las áreas de cartera, rehabilitación, normalización, funcionarios y educación financiera representando un $31 \%$ de la población atendida equivalente a (35 personas).

Los convenios empresariales por medio de Comfandi concentraron el $62 \%$ del total de las personas en la ruta de consultorios financieros (71 personas distribuidas en las siguientes instituciones: Industrias Culturales, Formas y Colores S.A.S. y Manuelita S.A.) Siendo el fuerte de la atención. Por otro lado, el de menor peso porcentual (\%) por no contar con un espacio por fuera de las instalaciones de la compañía fue el público particular, este represento $7 \%$ de la población total (8 personas).

El mayor índice de inasistencia lo presentó en la ruta interna con el área de rehabilitación generando 17 faltas es decir el 20\% de la población. En la externa, se generó por Industrias Culturales con el $14 \%$ de la misma.

En el diseño de la prueba piloto inicialmente se plantean tres sesiones, sin embargo con el transcurso del tiempo se tomó la decisiones modificar el proceso que se había realizado de tres sesiones con el Ingenio Manuelita y por efectos de desarrollo del mismo se reducen a dos sesiones; la primera presencial en donde se realiza el levantamiento de la información y la segunda de seguimiento que no fue obligatoria.

Además, cabe mencionar que el 95\% del público atendido fue el servicio de orientación financiera (Dr. Financiero) y el 5\% por el servicio de información; debido a las características demandadas de los clientes solo funciona la línea base de intervención del Dr. Financiero con el servicio de orientación financiera.

\subsection{SINAPSIS DEL CASO}

Los campos de destinación del caso presentado son Universidades, Empresas y todos los relevantes al sector financiero mundial, haciendo especial énfasis en las microfinanzas como lo soporta las teorías revisión de lectura presentada en esta ponencia modalidad casos.

Interés Social: Dado que hoy en día las personas están altamente vulnerables por el desconocimiento de su propia realidad financiera, hecho que conduce en últimas a la descomposición del tejido social y al surgimiento de actividades Microempresariales con altos costos en la obtención de crédito para el apalancamiento financiero de sus negocios. 
El interés Científico: dado que se analiza desde el método económico, el comportamiento de los individuos, frente a la toma de decisiones entre ellas, el uso eficiente de los recursos escasos y la restricción presupuestal, estos supuestos se contrastan con las teorías microeconómicas, como la teoría del consumidor y las teoría del mercado, mostrando en ultimas, el comportamiento y las preferencias de las personas para la satisfacción de sus necesidades, representadas en hechos económicos y estos últimos, se reflejan en fenómeno encaminados a la actividad económica microempresarial y el fortalecimiento de los estudiantes vinculados a los Programas académicos que ofrece la EMAVI (Escuela Militar de Aviación Marco Fidel Suarez de la Fuerza Aérea, en los componentes Organizacionales, Económicos y Financieros.

\subsection{OBJETIVOS PEDAGÓGICOS DEL CASO}

Este apartado busca que las diferentes instituciones Universitarias fortalezcan sus conocimiento en el tema, Económico y Financiero con el objetivo de que los estudiantes que se forman como profesionales, tengan unas competencias idóneas y puedan direccionar y administrar eficientemente las instituciones o áreas Financieras de las organizaciones, haciendo uso adecuado de esta herramienta con el fin de ayudar a contribuir en el fortalecimiento de las capacidades financieras de todas las personas en el mundo, entendiendo que estas pueden ser clientes internos y externos.

\subsection{DisCUSIÓN}

El tema de la inclusión financiera ha sido de interés nacional e internacional, comprendiendo que el acceso al capital permite superar condiciones de pobreza, igualmente permite mejorar la capacidad productiva de las micro, pequeñas y medianas empresas. Igualmente, al lado de una mayor bancarización de la población es necesario que se insista en el tema de la educación financiera, para que el manejo que se haga de los recursos financieros sea razonable, de tal manera que el empresario y/o ciudadano del común pueda tomar decisiones frente a los productos financieros que posee o espera poseer.

\section{CONCLUSIONES}

El fortalecimiento de las capacidades Financieras, económicas y Organizacionales que se lideran desde los claustros académicos con técnicas y metodologías de investigación, permite analizar los proceso de intervención social realizado por una Institución MF orientada a la 
educación financiera, esta demostró ser viable porque respondió a las características específicas de sus clientes, en cuanto contempló las realidades sociales y económicas, y el contexto del sector donde se tiene influencia. La metodología seguida para la construcción de la propuesta de intervención nació del análisis interno y externo, se tuvo en cuenta los lineamientos legales como los definidos por la Ley 1328 de 2009, lo dispuesto por ASOBANCARIA, y los intereses de aliados estratégicos. Así mismo, se consideró aspectos socio demográfico de la población de Cali, en especial la que se ubica en el rango de bajos ingresos. La estrategia local de intervención se caracterizó por contar con un modelo incluyente, que se ajusta a las condiciones de la población, y que promueve el desarrollo empresarial, en especial en el fortalecimiento de las unidades productivas del sector.

\section{REFERENCIA}

RODRÍGUEZ GARCÉS Catalina. (2008) Impacto de las microfinanzas: resultados de algunos estudios. Énfasis en el Sector. Financiero Revista Ciencias Estratégicas, vol. 16, núm. 20, juliodiciembre, pp. 281-298, Universidad Pontificia Bolivariana. Colombia.

OTERO MARÍA. (Ed.). (1998) El nuevo mundo de las finanzas microempresariales: estructuración de instituciones financieras sanas para los pobres, pp.339, Editorial Plaza y Valdes,

MARTINEZ Vásquez, Sergio A,(1997) Director de Saldebas, El nuevo Mundo de las Finanzas Microempresariales Julio de 1997

GÓMEZ Zamudio, Nancy, (2012) Las capacidades financieras de la población colombiana. Borradores de Economía No. 725, Banco de la República.

CAVADA ROBLES Daniel. (2002) Evaluación del área del Microcrédito un Techo para Chile. Centro de Investigación Social -CIS-. Santiago de Chile

AROCA, Patricio A. (2002) Estudio de programas de microcrédito en Brasil y chile, Urbana, Illinois, USA.

OSTROM, GARDNER Y WALKER ( 1994) “Rules, games and common-pool resources”, Ann Arbor, Estados Unidos, University of Michigan

OLIVARES Mirtha. (1989) Una metodología de trabajo con el sector informal (1989) Disponible en: http://centerforfinancialinclusionblog.files.wordpress.com/2011/09/accioninternationalaitec-a-methodology-for-working-with-the-informal-sector-spanish.pdf

MUÑOZ Lozano, Maribel: (2007) artículo La eficiencia del personal en las microfinanacieras influye en la cartera vencida, la Red de revistas científicas de América Latina y el Caribe, Universidad Autónoma del estado de México, ISSN: 0185-3937, disponible en http://redaly.uaemex.mx 